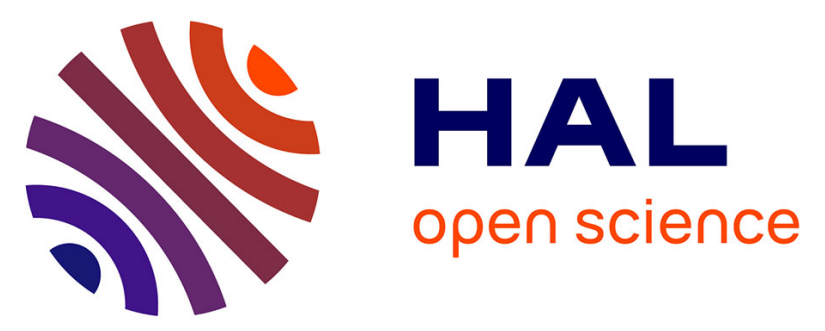

\title{
Elimination du bore dans le silicium par fusion de zone sous plasma inductif haute fréquence: rôle des plasmas réactifs et du laitier. Caractérisation du silicium photovoltaïque
}

\author{
D. Morvan, J. Amouroux, M. C. Charpin, H. Lauvray
}

\section{To cite this version:}

D. Morvan, J. Amouroux, M. C. Charpin, H. Lauvray. Elimination du bore dans le silicium par fusion de zone sous plasma inductif haute fréquence: rôle des plasmas réactifs et du laitier. Caractérisation du silicium photovoltaïque. Revue de Physique Appliquée, 1983, 18 (4), pp.239-251. 10.1051/rphysap:01983001804023900 . jpa-00245088

\section{HAL Id: jpa-00245088 https://hal.science/jpa-00245088}

Submitted on 1 Jan 1983

HAL is a multi-disciplinary open access archive for the deposit and dissemination of scientific research documents, whether they are published or not. The documents may come from teaching and research institutions in France or abroad, or from public or private research centers.
L'archive ouverte pluridisciplinaire HAL, est destinée au dépôt et à la diffusion de documents scientifiques de niveau recherche, publiés ou non, émanant des établissements d'enseignement et de recherche français ou étrangers, des laboratoires publics ou privés. 


\title{
Elimination du bore dans le silicium \\ par fusion de zone sous plasma inductif haute fréquence : rôle des plasmas réactifs et du laitier. Caractérisation du silicium photovoltaïque
}

\author{
D. Morvan, J. Amouroux, M. C. Charpin et H. Lauvray (*) \\ Ecole Nationale Supérieure de Chimie de Paris, Laboratoire de Génie Chimique, Equipe Réacteur en Phase Plasma, \\ 11, rue Pierre et Marie Curie, 75231 Paris Cedex 05, France \\ (*) Photowatt International S.A., 6, rue de la Girafe, 14001 Caen, France
}

(Reçu le 27 décembre 1982, accepté le 14 janvier 1983)

\begin{abstract}
Résumé - L'élaboration du silicium photovoltaïque exige de savoir contrôler de façon permanente sa pureté chimique et ses propriétés physiques. Les nombreuses études développées au cours des dernières années ont montré le rôle critique de certaines impuretés. Toutefois les travaux sur les associations chimiques capables d'apparaitre au cours de l'histoire thermique du matériau font défaut. Ainsi n'est-il pas alors étonnant de constater que les propriétés photovoltaïques d'un même matériau semblent dépendre des phases d'élaboration de la structure cristalline (C.Z., Bridgman, H.E.M....) [1-6], les impuretés subissant des ségrégations dans la phase liquide, aux joints de grain ou dans des combinaisons chimiques avec les laitiers de protection des creusets.

Parmi les techniques d'élaboration du silicium photovoltaïque nous avons cherché à développer la méthode originale de fusion de zone sous plasma inductif d'argon $[5,6]$. La technique qui s'apparente plus à une extraction conjointe solide-liquide, liquide-liquide et liquide-gaz permet l'élaboration à partir du silicium métallurgique d'un silicium polycristallin à gros grain et de très haute pureté.
\end{abstract}

\begin{abstract}
Preparation of photovoltaic silicon requires to analyse systematically the purity and physical properties of the material. Numerous studies developped during the last year show the critical effect of a number of impurities which affect the efficiency of the photocell. Nevertheless there still exists a gap in the studies concerning the chemistry combination which can occur between the impurities appearing during all technic operations of the material. Therefore it is clear that the photovoltaic properties of the same material seem to depend on the different steps of elaboration of a given crystallized structure (C.Z., Bridgman, H.E.M.) [1-6]. Indeed, impurities segregate in the liquid concentrate towards the grain boundaries or react with the protection slag of the crucible giving new chemical boundaries.

Among technics of the preparation of photovoltaic silicon we have chosen to develop the original method of purification of metallurgical grade silicon with a reactive plasma process $[5,6]$. This process which is in fact a liquid-solid, liquid-liquid and liquid-gas extraction permits to obtain a polycrystalline silicon having a high purity. with crystals measuring $1 \mathrm{~mm}$ or slightly more.
\end{abstract}

1. Techniques d'analyse permettant de caractériser et contrôler l'élaboration du silicium. - L'analyse chimique totale et précise du silicium n'est pas chose facile. Les 107 éléments ne sont pas dosables à la même sensibilité par toutes les techniques et il faut admettre qu'une analyse atomique doit être complétée par une analyse de type IR ou ESCA pour identifier les liaisons chimiques entre éléments. Enfin, les analyses physiques (EBIC, résistivité, rendement de photopiles) établissent les propriétés physiques du matériau et ses corrélations avec la teneur en impureté ou l'importance des combinaisons chimiques.
1.1 Etude de la CARACtérisation PHysiQue du SILICIUM POlyCRISTAllin. - Pour un monocristal de silicium la résistivité est un paramètre important qui caractérise le matériau de base d'une cellule photovoltaïque. Elle dépend de la concentration et de la distribution des impuretés, ainsi que des défauts cristallins (Fig. 1). Dans un polycristal [7-10] les cristaux d'orientation et de dimensions variables sont séparés les uns des autres par des zones perturbées où des ségrégations d'impuretés peuvent avoir lieu : les joints de grain. Ces derniers agissent comme des pièges pour les porteurs et modifient les caractéristiques électriques 


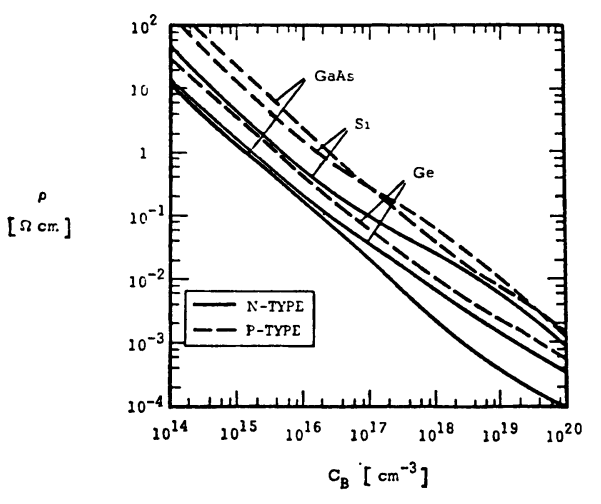

Fig. 1. - Variation de la résistivité avec la concentration en bore (Réf. [27]).

[Influence of boron concentration on resistivity.]

de l'échantillon. Une étude montre [11] que dans le cas des polycristaux à gros grains et de haute pureté l'influence des joints de grain sur les mesures physsiques telles que la résistivité, est relativement faible. Le joint de grain serait assimilé à une ligne de dislocation et son influence diminuerait encore lorsque les grains seraient orientés dans la même direction [12].

Les mesures physiques sont sensibles par contre non seulement à la concentration en élément dopant (B, P) mais également à des impuretés présentes même en faible quantité [13]. Actuellement il n'existe pas de modèles satisfaisants pour prévoir et comprendre le comportement de l'ensemble des impuretés. La présence d'impuretés crée des niveaux d'énergie supplémentaires et deux cas typiques peuvent être présentés.

1) Les niveaux supplémentaires sont situés au voisinage immédiat des bords des bandes de valence et de conduction, ce sont des niveaux dits superficiels et ils ont alors un rôle de dopant.

2) Le second cas est celui des niveaux profonds proches du milieu de la bande interdite qui peuvent jouer le rôle actif (donc très néfastes dans les photopiles) de centres de recombinaison.

En fait ce schéma reste théorique et il semble difficile de préciser le caractère dopant, neutre, ou centre recombinant de tous les éléments.

La mesure de résistivité par la méthode de spreading résistance ou par celle des 4 pointes est depuis longtemps utilisée dans l'industrie électronique pour caractériser le silicium dopé au bore [14]. Des études montrent [7-11] que la mesure reste valable pour un polycristal de silicium lorsque les grains sont de taille importante $\left(\simeq 3 \mathrm{~mm}^{2}\right)$. Cette méthode permet souvent la mesure de la teneur en bore ou plus généralement de la somme des impuretés dopantes.

Le contrôle des défauts électriquement actifs : impuretés, dopants, joints de grain et joints de macle peuvent être mesurés par microscopie électronique à balayage en mode EBIC. Dans le cas d'un polycristal cette mesure est particulièrement intéressante puis- qu'elle permet d'identifier le défaut physique à l'origine de la perturbation.

Enfin la réalisation des cellules solaires [15] permet de mesurer le rendement photovoltaïque et la longueur de diffusion c'est-à-dire de définir les qualités photovoltaïques du matériau.

1.2 Analyses Chimiques. - Afin de contrôler l'évolution chimique du silicium lors de son élaboration il est indispensable de disposer de plusieurs techniques analytiques.

Dans ce but nous avons retenu les 6 techniques analytiques suivantes :

- l'analyse par spectrométrie de masse à étincelle,

- l'analyse par radioactivation,

- l'analyse par spectrométrie d'émission plasma, inductif haute fréquence,

- l'analyse ESCA,

- l'analyse par rayon $\mathrm{X}$,

- l'analyse par spectrométrie d'absorption infrarouge.

1.2.1 Analyse par spectrométrie de masse à étincelle. - Cette technique a une grande sensibilité pour les éléments monoisotopiques. Elle présente l'avantage d'indiquer à des teneurs comprises entre la ppb et la ppm des concentrations en éléments difficilement accessibles par d'autres techniques (B, C, F, $\mathbf{P}, \mathbf{S})$.

Néanmoins dans le cas d'un matériau polycristallin les impuretés se ségrègent principalement aux joints de grain. Nous savons par ailleurs que l'étincelage a lieu préférentiellement dans ces zones hétérogènes conductrices. Ainsi la reproductibilité de l'analyse dépend de la localisation des impuretés de sorte que dans le cas d'un silicium polycristallin la mesure effectuée indique généralement une teneur en impureté supérieure à la concentration moyenne de l'échantillon.

1.2.2 Analyse par radioactivation. - Elle permet une analyse élémentaire très générale et se présente comme une technique complémentaire de l'analyse par spectrométrie de masse. Certes, les informations concernant les éléments $B, C, F, P, S$ dans le silicium pur ne peuvent être données par la radioactivation mais pour les impuretés métalliques et notamment les métaux de transition la limite de détection se situe entre $1 \mathrm{ppm}$ et $0,1 \mathrm{ppb}$ selon l'élément, la masse analysée et les conditions d'irradiation choisies. Dans le cas du silicium photovoltaïque des éléments tels que le fer, le chrome, le titane et le zirconium et le zinc ne peuvent être dosés aux faibles concentrations $(<\mathrm{ppm})$ qu'après une irradiation prolongée de 3 jours.

1.2.3 Analyse par plasma. - Afin de compléter l'analyse élémentaire du silicium nous avons développé au laboratoire l'analyse par spectrométrie d'émission plasma inductif. La qualité du matériel spectrométrique et les caractéristiques de la torche plasma rendent possible l'analyse des éléments $\mathrm{Ca}$, 
$\mathrm{Cu}, \mathrm{Mn}, \mathrm{Al}$ et $\mathrm{B}$ à des concentrations voisines de la $\mathrm{ppb}$. Cette technique complète les deux précédentes puisque pour ces éléments nous obtenons de meilleures limites de détection. De plus nous pouvons doser des éléments à faible concentration tels que le bore et le calcium.

1.2.4 Analyse ESCA. - L'analyse consiste à déterminer les énergies des niveaux électroniques par la mesure à haute résolution de l'énergie cinétique des photoélectrons émis par l'échantillon à analyser. Ces photoélectrons sont générés par des processus photoélectriques sous irradiation de photons d'énergie connue. Les caractéristiques principales de l'analyse ESCA sont :

- analyse non destructive, $100 \AA$,

- une analyse de surface sur une profondeur de

- une analyse à la fois qualitative et quantitative.

Enfin si les techniques analytiques précédentes permettaient de connaitre la concentration élémentaire des impuretés avec l'ESCA il est possible d'identifier la nature des associations chimiques qui peuvent apparaître entre les éléments et d'en préciser l'importance en fonction du traitement subi par l'échantillon.

Expérimentalement la mesure précise de l'énergie de liaison permet de mettre en évidence l'évolution de la liaison en fonction du traitement thermique et chimique du matériau.

Ainsi cette analyse indique pour un environnement chimique imposé par le traitement de préciser comment les combinaisons chimiques se sont édifiées et de conclure sur la façon d'opérer pour éliminer une impureté comme le bore par exemple.

1.2.5 Analyse par diffraction des rayons $\mathrm{X}$ des laitiers de purification. - L'identification d'un corps pur ou des composants d'un mélange à l'aide de la diffraction des rayons $X$ est couramment utilisée en métallurgie. La méthode de Debye Scherrer consiste à soumettre un faisceau de rayons $X$ sur un échantillon pulvérisé et d'enregistrer les raies diffractées. L'interprétation du diagramme permet de mesurer les distances réticulaires et ainsi de caractériser à l'aide de tables les composés présents dans l'échantillon. Cette technique permet de caractériser des composés complexes dont la teneur est supérieure à $5 \%$; elle s'adapte bien à l'analyse des laitiers de surface employés pour purifier le silicium. Elle permet de comprendre sous quelle forme chimique les impuretés auront été piégées dans celui-ci.

1.2.6 Analyse par spectrométrie d'absorption infrarouge. - La spectrométrie d'absorption infrarouge est utilisée en analyse qualitative et quantitative des liaisons d'un composé minéral ayant cristallisé. En choisissant les conditions analytiques précises il est notamment possible d'obtenir une corrélation simple entre l'absorption de l'oxygène dans le silicium et la concentration de cette impureté [16]. A partir du rapport des coefficients d'absorption obtenus à l'am- biante et à la température de l'hélium liquide on peut doser l'oxygène dans le silicium avec une limite de détection très faible $(1 \mathrm{ppb})$. Cette analyse complète l'analyse ESCA et présente l'avantage d'être une mesure dans la masse de l'échantillon. L'oxygène présent dans le silicium peut migrer ou précipiter durant les traitements thermiques. Le traitement sous plasma est donc susceptible de modifier la concentration en oxygène dans l'échantillon et par voie de conséquence de provoquer une modification de ses propriétés électriques (résistivité, rendement de photopile).

2. Technique choisie pour l'élimination du bore dans le silicium - La technique de fusion de zone sous plasma choisie consiste à fondre localement une barre de silicium par l'emploi d'un plasma inductif haute fréquence [5-6].

L'installation du laboratoire, photo 1 , comporte quatre spires inductives en cuivre refroidies à l'eau qui assurent l'ionisation du gaz plasmagène. Cet inducteur est alimenté par un générateur haute fréquence $13 \mathrm{MHz}$ et d'une puissance variable de 3 à $12 \mathrm{~kW}$. Les spires entourent le' tube en quartz dans lequel circule le gaz. L'étanchéité entre ce tube de quartz et la mécanique de fusion de zone est assurée par un rodage sphérique. La mécanique de fusion de zone

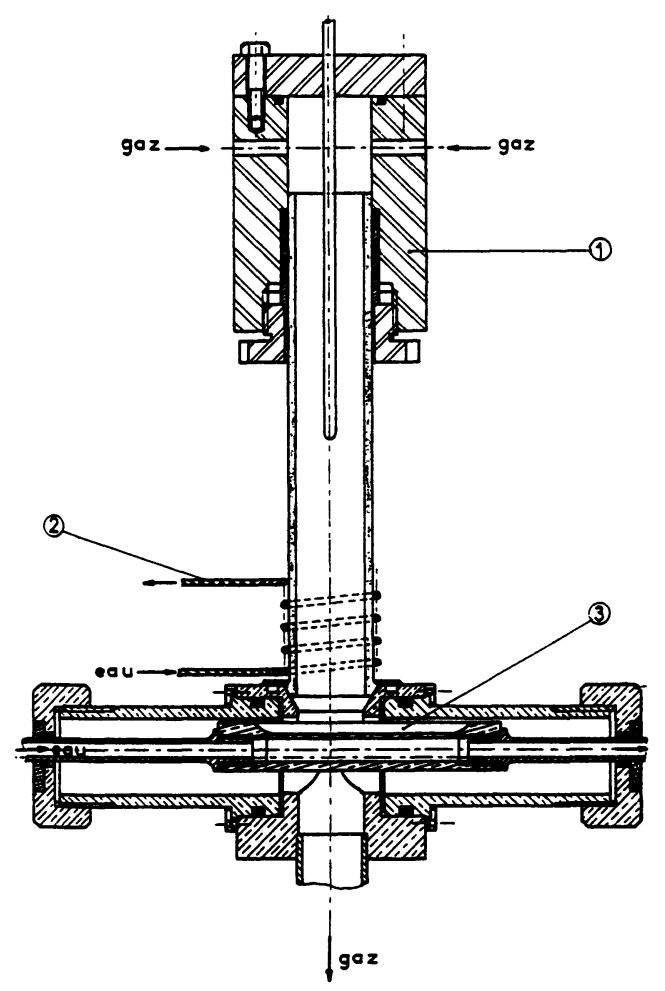

Photo 1a. - Installation de fusion de cône sous plasma : (1) Tête d'amenée du fluide plasmagène $\left(\mathrm{Ar}+\mathrm{H}_{2}+\mathrm{O}_{2}\right)$; (2) Inducteur d'alimentation électrique $(13 \mathrm{MHz}, 3$ à $12 \mathrm{~kW}$ ); (3) Nacelle refroidie.

[Installation of zone melting by plasma : (1) Inlet of plasma gas $\left(\mathrm{Ar}+\mathrm{H}_{2}+\mathrm{O}_{2}\right)$; (2) Inductor of electric feeding (13 $\mathrm{MHz}, 3$ to $12 \mathrm{~kW}$ ); (3) Cooled copper crucible.] 


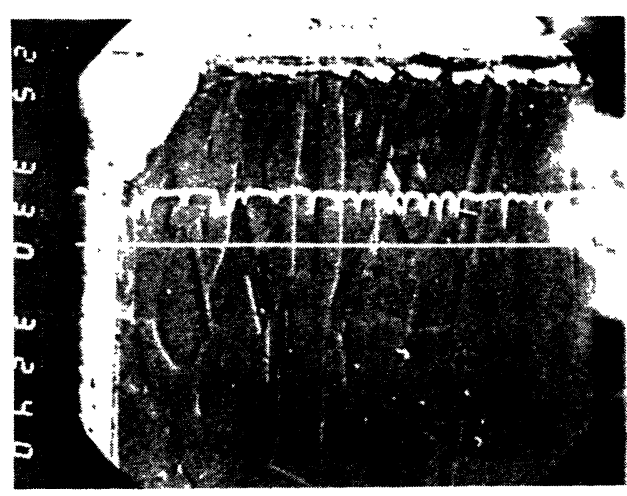

Photo $1 b$. - Mesure EBIC sur un échantillon purifié par fusion de zone sous plasma.

[EBIC mesure on a purified sample by plasma zone melting process.]

sous plasma est en cuivre refroidi à l'eau. A la verticale du tube de quartz une ouverture permet l'évacuation des gaz. Une nacelle de cuivre refroidie se déplace horizontalement et supporte le barreau à traiter. L'ionisation du gaz provoque l'apparition d'une torche à plasma qui vient fondre l'échantillon localement Cette zone liquide se déplace de la tête vers la queue du barreau. Le fluide plasmagène est constitué par un mélange argon-hydrogène-oxygène. La teneur en hydrogène est voisine de $1 \%$ et la teneur en oxygène est comprise entre $0,2 \%$ et $0,01 \%$ selon la quantité de bore à éliminer. Le traitement consiste à choisir les conditions opératoires et notamment la vitesse et le nombre de passages sous le jet de plasma afin de pouvoir optimiser la fusion et l'élimination des impuretés et notamment du bore.

Cette technique présente comme particularité d'effectuer une fusion en auto-creuset, de cette façon aucune impureté provenant de la nacelle n'est introduite en cours de traitement. La fusion a lieu à une pression légèrement supérieure à la pression atmosphérique n'occasionnant de fait aucun problème d'étanchéité.

Il s'agit donc d'une technique non polluante puisque les gaz utilisés sont ultra-purs et le plasma est généré par une méthode inductive donc sans électrode. Cette technique enfin présente de nombreuses différences avec la fusion de zone classique(Tableau I).

3. Mise en évidence de l'élimination du bore dans le silicium par fusion de zone sous plasma. Etude expérimentale. - 3.1 CHOIX DES ÉCHANTILLONS DE DÉPART ET DES CONDITIONS EXPÉRIMENTALES. - Le but de notre travail est de mesurer l'élimination du bore dans le silicium par fusion de zone sous plasma réactif. Pour cela nous avons choisi de comparer la concentration initiale et finale du silicium électronique dopé au bore. Les échantillons de départ sont monocristallins (C.Z.) et ultra-purs. Deux types d'échantillons ont été testés :
Tableau I. - Comparaison entre fusion de zone classique et fusion de zone sous plasma.

[Comparison between classical zone melting and plasma zone melting process.]

\begin{tabular}{|c|c|c|}
\hline Parametres: & Fusion classique & fusion sous plasma \\
\hline Materiau de depart & pur 99,9 . & Impux 99,5 ou 98 , \\
\hline Nombre de phase en presence & 2: solide liquide & $\begin{array}{l}\text { 4.: solide, liquide, } \\
\text { laitier, gaz plasma }\end{array}$ \\
\hline vitesse de traitement & 1 $50 \mathrm{~mm} / \mathrm{h}$ & 400 a $1200 \mathrm{~m} / \mathrm{h}$ \\
\hline $\begin{array}{l}\text { Temperature de la goutte } \\
\text { inquide. }\end{array}$ & $1800-1900^{\circ} \mathrm{C}$ & $2300-2500^{\circ} \mathrm{C}$ \\
\hline $\begin{array}{l}\text { Coefficient de partage } \\
\text { effectif }\end{array}$ & $\begin{array}{c}10^{2} \text { a } 10^{4} \text { le } \\
\text { coeff } 2 \text { icient } a_{1} \cdot \text { equilibre }\end{array}$ & $\begin{array}{l}10^{2} \text { \& } 10^{-2} \text { le coef- } \\
\text { ficient a } 1 \text { \&quilit- } \\
\text { bre entre } 2 \text { phases }\end{array}$ \\
\hline Nombre de passes & 10 à 50 & 4 \\
\hline Impuretés & queue & $\begin{array}{l}\text { queue et en surface } \\
\text { du barreau }\end{array}$ \\
\hline Sens du traitement & sens unique téte $\rightarrow$ queue & tête $\mp$ queue \\
\hline Gaz environnant & frold $\left(100-500^{\circ} \mathrm{C}\right)$ & chaud $8000-9000^{\circ} \mathrm{x}$ \\
\hline Réactivité du gaz & faible & $\begin{array}{l}\text { Ion1se done tress } \\
\text { reactif }\end{array}$ \\
\hline $\begin{array}{l}\text { Barridere thermique } \\
\text { de surface }\end{array}$ & out & non \\
\hline Laitier & non & out \\
\hline $\begin{array}{l}\text { Role de la diffusion } \\
\text { dans la purification }\end{array}$ & 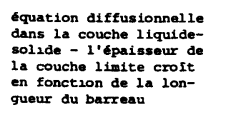 & $\begin{array}{l}\text { role de convection } \\
\text { dans la goutte } \\
\text { 12quide }\end{array}$ \\
\hline Evaporation an surface & faible & très importante \\
\hline $\begin{array}{l}\text { Coefficient de purification } \\
\text { globale apres } 4 \text { passes }\end{array}$ & $10-20$ & 10.000 \\
\hline $\begin{array}{l}\text { E1/mination des impuretes } \\
\text { a constante d'equilibre } \mathrm{X}=1\end{array}$ & non & $\begin{array}{l}\text { ouz par le role du } \\
\text { systeme multiphase }\end{array}$ \\
\hline Energie consommé & 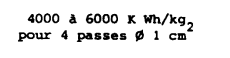 & $\begin{array}{l}50-100 \mathrm{KWH} / \mathrm{kg} \text { pour } \\
4 \text { passes } \varnothing 4 \mathrm{~cm}^{2}\end{array}$ \\
\hline Systeme & Creuset refroid2 & autocreuset \\
\hline
\end{tabular}

1) Le silicium dopé à $34 \mathrm{ppb}$ de bore soit $45 \times$ $10^{15}$ at $/ \mathrm{cm}^{3}$ et $\rho=1$ à $3 \Omega$.cm,

2) Le silicium dopé à $74 \mathrm{ppm}$ de bore soit $10^{17} \mathrm{at} /$ $\mathrm{cm}^{3}$ et $\rho=0,011 \Omega$.cm.

Ce choix nous a permis de prévoir pour des concentrations de départ en bore différentes comment établir les débits gazeux du plasma afin d'assurer dans les 2 cas une élimination du bore.

Le mode opératoire retenu [5-6] consiste à fixer la vitesse de déplacement du barreau à $40 \mathrm{~cm} / \mathrm{h}$ et à contrôler l'élimination du bore en fonction de la concentration en oxygène dans le plasma et du nombre de passage $[17,18]$.

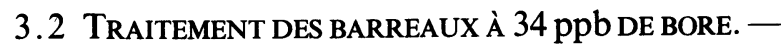
La série d'essais montre que deux passages sous un plasma d'argon-hydrogène avec $0,01 \%$ d'oxygène conduit à une augmentation de la résistivité de 1 à $5 \Omega . \mathrm{cm}$ tout au long du barreau, c'est-à-dire que la concentration en bore passe de $34 \mathrm{ppb}$ à $20 \mathrm{ppb}$. Une amélioration ultérieure peut être obtenue avec un plasma réducteur argon-hydrogène pour éliminer les défauts introduits par le plasma oxygéné. Avec un plasma non oxygéné l'élimination du bore a également lieu et ceci est probablement dû à l'oxygène résiduel (10 ppm) contenu dans l'argon pur.

Le traitement effectué provoque l'apparition de défauts structuraux importants puisque, à partir d'un monocristal, nous obtenons un polycristal avec des grains de 1 à $5 \mathrm{~mm}$ orientés du centre du barreau 
vers la surface. Malgré ce changement cristallographique la résistivité reste améliorée.

Avec deux échantillons traités dans ces conditions nous avons réalisé des photopiles (Tableau II) dont le rendement photovoltaïque se situe entre $3,7 \%$ et $8 \%$ sans couche anti-reflet. L'interprétation de l'ensemble des mesures montre que la grosseur des grains du polycristal est probablement à l'origine de ce résultat. Ainsi pour l'échantillon à gros grains $\left(3 \mathrm{~cm}^{2}\right)$ la valeur du rendement est élevé 6,6 à $8 \%$ avec une longueur de diffusion qui est passée de 100 à $150 \mu \mathrm{m}$ au départ, à $50 \mu \mathrm{m}$ après traitement. Pour l'échantillon à petits

Tableau II. - Résultats du rendement des photopiles et mesures physiques.

[Results of physical measure and efficiencies of the cells.]

\begin{tabular}{|c|c|c|c|c|c|}
\hline $\begin{array}{l}\text { Echuntillon } \\
\text { de depart }\end{array}$ & 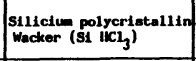 & 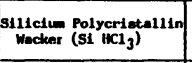 & $\begin{array}{l}\text { S11Hcilum mono } \\
1-3 \cap \mathrm{C}\end{array}$ & $\underset{1-3 \Omega \text { silicium mono }}{\substack{1 \\
\text { mon }}}$ & $\begin{array}{l}\text { Silicium metallurqi - } \\
\text { que } 99,7 \times\end{array}$ \\
\hline Traitement & c.z. & F.2.P. & F.z.p. & F.z.P. & F.7.P. \\
\hline Echantillon obtenu & Monocristallin & Polycristallin & Polycriatallin & Polycristallin & Polycristallin \\
\hline 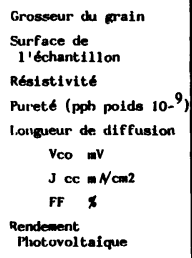 & $\begin{array}{c}78 \mathrm{~cm} 2 \\
78 \mathrm{~cm} 2 \\
1-3 \Omega \mathrm{cm} \\
20-100 \mathrm{ppb} \\
100-150 \mu \mathrm{m} \\
575-600 \mathrm{mv} \\
26,7-29,3 \mathrm{~m} / \mathrm{cm} 2 \\
0,75 \\
11,8-14,5 x\end{array}$ & $\begin{array}{l}1-3 \mathrm{~m} 2 \\
2 \mathrm{~cm} 2 \\
100 \Omega \mathrm{cm} \\
45 \mu=\end{array}$ & $\begin{array}{c}0,5 \mathrm{~cm} 2 \\
2 \mathrm{~cm} 2 \\
3-5 \cap \mathrm{cm} \\
20-100 \mathrm{ppb} \\
50 \mu \mathrm{m} \\
470-490 \mathrm{mV} \\
20-23 \mathrm{~mA} / \mathrm{cm} 2 \\
0,70 \\
6,6-8 x\end{array}$ & $\begin{array}{c}1-15 \mathrm{~m} 2 \\
2 \mathrm{~cm} \\
2-3,5 \cap \mathrm{cm} \\
20-100 \mathrm{ppb} \\
10-50 \mu \mathrm{m} \\
425-501 \mathrm{mV} \\
12,5-17,5 \mathrm{mN} / \mathrm{cm} 2 \\
0,70 \\
3,7-6,1 \times\end{array}$ & $\begin{array}{c}1-15 \mathrm{~mm} 2 \\
2,5 \mathrm{~cm} 2 \\
0,5-5 \Omega \mathrm{cm} \\
50-100 \mathrm{pph} \\
20-30 \mu \mathrm{m} \\
335-390 \mathrm{mv} \\
13,5-14,9 \mathrm{~mA} / \mathrm{cm} ? \\
0,60-0,65 \\
2,7-3,7 \times\end{array}$ \\
\hline & $\begin{array}{c}\text { Taux do rocouvreanent } \\
10 x\end{array}$ & & $\begin{array}{l}\text { Echantill ons brute } \\
\text { couctio an }\end{array}$ & $\begin{array}{l}\text { recouvrement } 20 \% \\
\text { usion sous plasme san } \\
\text { Plet }\end{array}$ & so dopegen - sans \\
\hline
\end{tabular}

grains $\left(0,5\right.$ à $\left.1,5 \mathrm{~cm}^{2}\right)$ la longueur de diffusion est hétérogène et varie de 10 à $50 \mu \mathrm{m}$, le rendement est alors de 3,7 à $6,1 \%$.

Soulignons toutefois que le matériau ainsi traité reçoit un choc thermique lié à la fusion plasma aussi les contraintes et les défauts cristallographiques résiduels sont importants. Ce résultat est mis en évidence par l'EBIC qui indique notamment qu'un joint de macle perturbe autant le signal que le joint de grain dans le cas d'un traitement de fusion sous plasma (Photo 1).

Tableau III. - Analyse des différents silicium métallurgiques (PUK) par radioactivation (C.E.C.M.), spectrométrie de masse $夫$ (C.G.E.).

[Analysis of different metallurgical grade silicon by radioactivation, mass spectrometry $夫$. Results in ppm weight.]

\begin{tabular}{|c|c|c|c|}
\hline \begin{tabular}{|} 
coefficient de \\
distribution $X_{0}$
\end{tabular} & Impuretess & $\begin{array}{l}\text { Silicium metallurgique } \\
11(98 \text { x) }\end{array}$ & $\begin{array}{l}\text { Silicium metallurgique } \\
2(99,78)\end{array}$ \\
\hline 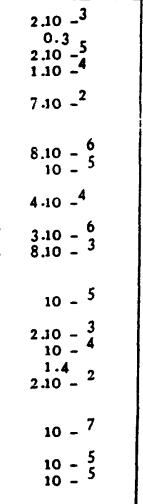 & 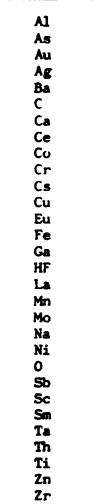 & $\begin{array}{c}3000 \\
<0,08 \\
0,2 \\
20 \\
1900 \\
2000 \\
5 \\
40 \\
40 \\
1800 \\
1,5 \\
10 \\
43 \\
10 \\
7,8 \\
80 \\
4000 \\
0,07 \\
0,62 \\
0,002 \\
160 \\
40 \\
0,3\end{array}$ & 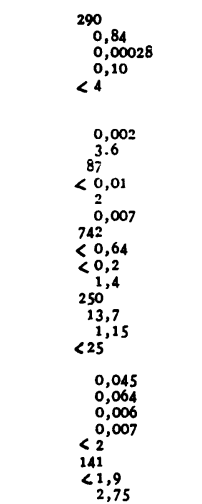 \\
\hline
\end{tabular}

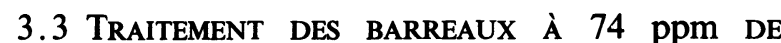
BORE. - Ces échantillons ont une concentration très élevée en bore, de ce fait la teneur en oxygène introduit dans le plasma d'argon joue un rôle déterminant pour l'élimination du bore. Sur la figure 2 nous

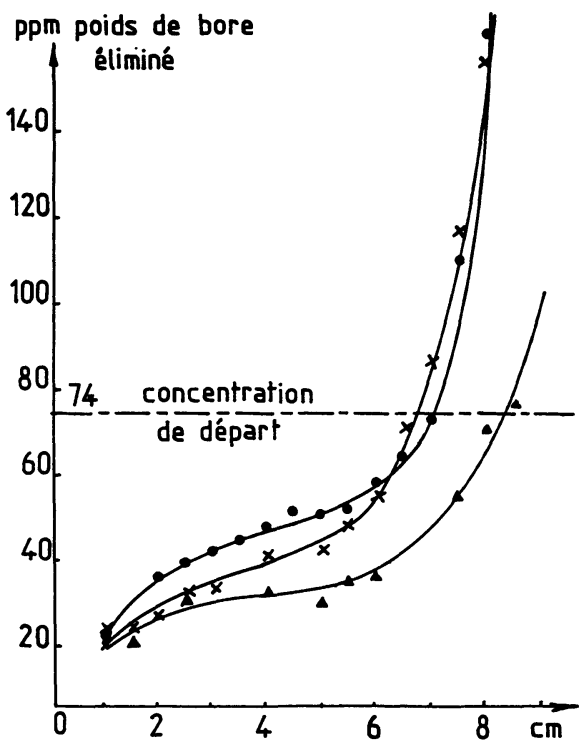

Fig. 2. - Evolution de la teneur en bore éliminée en fonction de la distance par rapport à la tête de barreau et de la quantité d'oxygène dans le plasma. Vitesse de déplacement : $40 \mathrm{~cm} / \mathrm{h}$, débit d'argon : $35 \mathrm{l} / \mathrm{min}$., débit d'hydrogène : $70 \mathrm{cc} / \mathrm{min}$., débit d'oxygène : $2,5 \mathrm{cc} / \mathrm{min} ., \times 90 \mathrm{cc} / \mathrm{min}$., $\Delta 60 \mathrm{cc} / \mathrm{min}$.

[Evolution of boron eliminated as a function of the length from the head of the bar and as a function of percentage of oxygen in the plasma. Rate of zone travel $: 40 \mathrm{~cm} / \mathrm{h}$, argon flow rate : $35 \mathrm{l} / \mathrm{min}$., hydrogen flow rate $: 70 \mathrm{cc} / \mathrm{min}$., oxygen flow rate : $2.5 \mathrm{cc} / \mathrm{min}$., $\times 9 \mathrm{cc} / \mathrm{min}$., $\triangle 60 \mathrm{cc} / \mathrm{min}$.] 
avons porté la concentration en bore en fonction de la distance par rapport à la tête du barreau. Nous constatons qu'après 4 passages à $40 \mathrm{~cm} / \mathrm{h}$ sous un plasma d'argon avec $0,2 \%$ d'hydrogène et $0,01 \%$ d'oxygène, seulement $15 \mathrm{ppm}$ de bore ont été éliminées. Avec une teneur en oxygène de $0,12 \%$ il est possible d'éliminer $40 \mathrm{ppm}$ de bore sur toute la partie purifiée. Ces courbes indiquent que la concentration en oxygène contrôle l'élimination du bore dans le silicium. Cette élimination du bore s'effectue de deux façons : d'une part par un drainage en surface de la goutte de liquide et en queue de barreau et d'autre part par volatilisation du bore ou des oxydes de bore sous l'action du plasma. A partir des courbes de la figure 2 le calcul (Fig. 3) permet de préciser le rôle de la con-

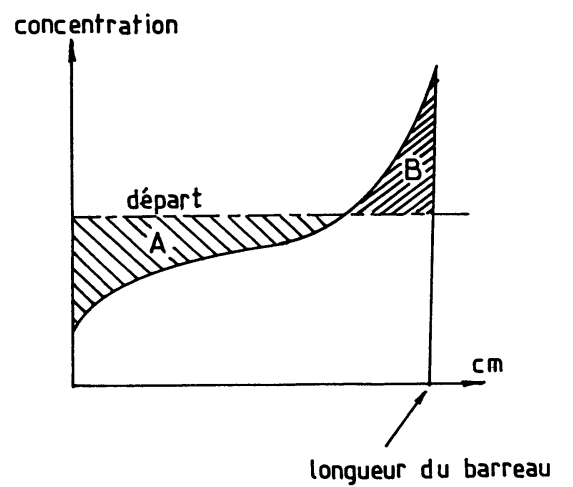

Fig. 3. - Principe du calcul de la quantité de bore éliminé et évaporé. A : quantité totale de bore éliminé dans la partie purifiée, B : quantité de bore concentrée en queue, A - B : quantité de bore évaporé.

[Principle of calculation of the quantity of boron eliminated and evaporated. A : Total quantity of boron eliminated in the purity part, B : Quantity of boron concentrated in the tail, A - B : Quantity of boron evaporated.]

centration en oxygène sur l'importance et le mode d'élimination du bore. La figure 4 résume les conclusions précédentes en indiquant la composition chimique du plasma à retenir afin d'éliminer une teneur définie en bore.

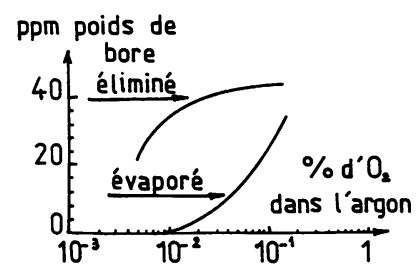

Fig. 4. - Elimination du bore par évaporation et drainage en fonction de la quantité d'oxygène dans le plasma. Echantillon de départ : silicium électronique $0,011 \Omega$. cm, c'est-àdire contenant $74 \mathrm{ppm}$ poids de bore. Résultats obtenus après 4 passages à $40 \mathrm{~cm} / \mathrm{h}$.

[Elimination of boron by evaporation and drainage as a function of percentage of oxygen in the plasma. Raw material : electronic silicon $(0.011 \Omega . \mathrm{cm})$ having $74 \mathrm{ppm}$ weight of boron. Results obtained after four pass at $40 \mathrm{~cm} / \mathrm{h}$.]
Par ailleurs l'élimination du bore augmente avec le nombre de passages en fusion de zone et quelle que soit la concentration en oxygène. Cette purification n'est pas asymptotique de sorte qu'une augmentation du nombre de passages peut conduire à une élimination totale du bore présent dans le barreau (Fig. 5).

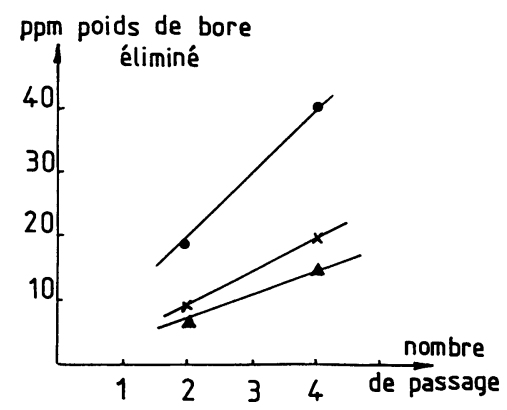

Fig. 5. - Elimination du bore dans les échantillons de silicium électronique $0,011 \Omega . \mathrm{cm}$ en fonction du nombre de passages et de la concentration en oxygène dans le plasma. Débit d'argon : $35 \mathrm{l} / \mathrm{min}$., débit d'hydrogène : $70 \mathrm{cc} / \mathrm{min}$., débit d'oxygène : $\Delta 2,5 \mathrm{cc} / \mathrm{min}$., $\times 9 \mathrm{cc} / \mathrm{min}$., $60 \mathrm{cc} / \mathrm{min}$.

[Elimination of boron in silicon electronic samples $0,011 \Omega . \mathrm{cm}$ as a function of the number of pass and the oxygen concentration in the plasma. Argon flow rate : $35 \mathrm{l} / \mathrm{min}$., Hydrogen flow rate : $70 \mathrm{cc} / \mathrm{min}$., Oxygen flowrate : $\triangle 2.5 \mathrm{cc} / \mathrm{min} . \times 9 \mathrm{cc} / \mathrm{min}$., $60 \mathrm{cc} / \mathrm{min}$.]

\subsection{INTERPRÉTATION SUR L'ÉLIMINATION DU BORE} À L'AIDE DE L'ANALYSE PAR SPECTROMÉTRIE D'ABSORPTION INFRAROUGE ET PAR ESCA. ANALYSE DES OXYDES DE BORE ET DE L'OXYGÈNE. - 3.4.1 Analyses ESCA. L'échantillon témoin de départ étant le silicium électronique ultra-pur dopé $\mathrm{P}^{+}(74 \mathrm{ppm}$ de bore soit $0,011 \Omega . \mathrm{cm}$ ), l'analyse ESCA (Fig. 6) montre que le bore utilisé comme dopant se trouve sous les trois formes suivantes :

1) Le bore lié au silicium constitue le dopant du matériau électronique. Il apparaît de façon précise sur le spectre ESCA à une énergie de $184 \mathrm{eV}$ pour le bore de type $\mathrm{B}_{y} \mathrm{Si}_{x}$ et nous pouvons constater que sa concentration est faible.

2) Le bore sous forme métallique apparaît à 186,6 eV. De degré d'oxydation nul il ne participe pas au dopage du matériau, sa concentration est voisine du bore lié au silicium.

3) Enfin, un bore oxydé apparaît à $189,9 \mathrm{eV}$, il correspond au degré d'oxydation de $\mathrm{BO}$ ou $\mathrm{B}_{2} \mathrm{O}_{2}$. Sa concentration est importante par rapport aux deux autres composés $\left(\mathrm{Si}_{x} \mathrm{~B}_{y}\right.$ et $\left.\mathrm{B}\right)$.

Ainsi l'analyse ESCA du matériau électronique dopé $\mathrm{P}^{+}$indique que le nombre d'atomes de bore intervenant dans le dopage du matériau est faible par rapport au nombre d'atomes de bore total présent dans la matrice silicium. Cette remarque est importante car elle peut expliquer l'écart constaté entre la concentration en bore déduite de la résistivité $\rho=f$ (concentration en atomes de bore) et d'une analyse 


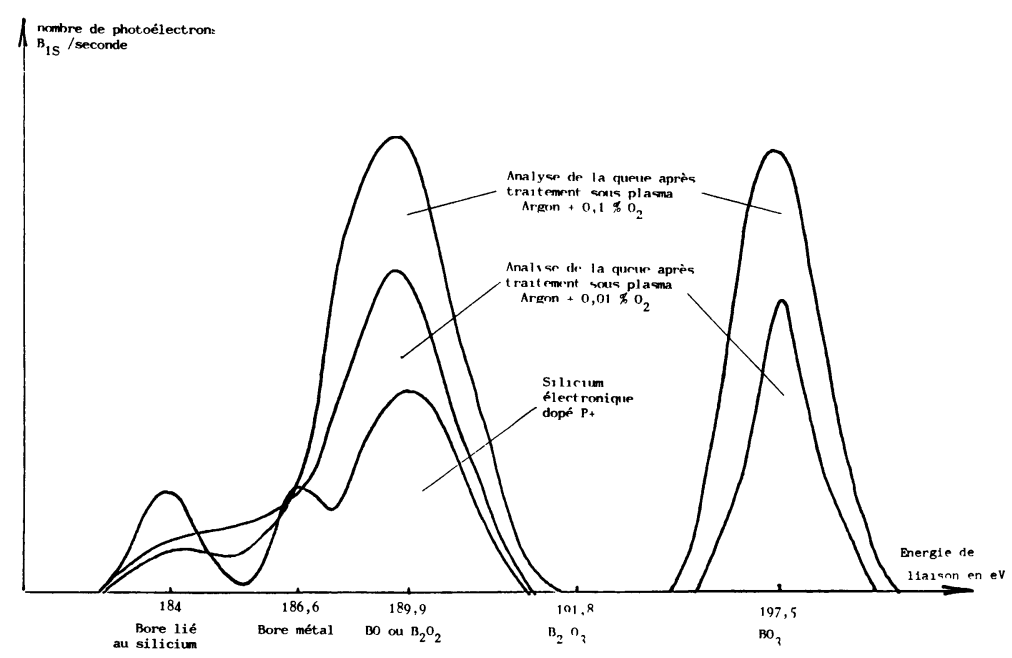

Fig. 6. - Analyse ESCA : mise en évidence de l'élimination du bore par un plasma oxygéné. Oxydation du bore lié au silicium. Concentration des oxydes en surface et en queue du barreau.

[ESCA analysis : Elimination of boron by an oxygened plasma. Oxidation of boron bounded with silicon. Concentration of oxides on the surface and at the tail of the bar.]

Tableau IV. - Evolution de la pureté en fonction du silicium métallurgique de départ. Résultats pour 4 passages à $40 \mathrm{~cm} / \mathrm{h}$ avec un traitement acide. Comparaison avec le silicium polycristallin Wacker. Résultats en ppm poids : analyse par radioactivation (C.E.C.M. Vitry), analyse par spectrométrie de masse (C.G.E.).

[Evolution of the purity as a function of the choice of metallurgical grade silicon. Results for four pass at $40 \mathrm{~cm} / \mathrm{h}$ with an acidic leaching. Comparison with polycrystalline Wacker silicon. Analysis with radioactivation, mass spectrometry. Results in ppm weight.]

\begin{tabular}{|c|c|c|c|}
\hline Impuret tes & $\begin{array}{l}\text { Purification obtenue } \\
\text { avec le s.11icium mé- } \\
\text { tallurgique } 1(98 \%) \text {. }\end{array}$ & 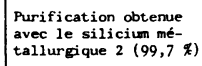 & 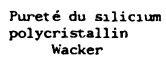 \\
\hline 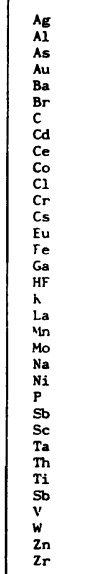 & $\begin{array}{l}<1 \\
0,00002 \\
0,9 \\
\\
0,008 \\
<0,02 \\
0,01 \\
<1 \\
0,015 \\
0,003 \\
\\
0,0001 \\
<0,0004 \\
0,001 \\
<0,07\end{array}$ & 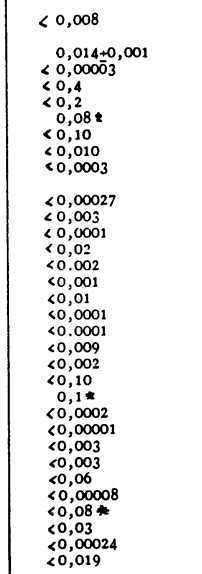 & 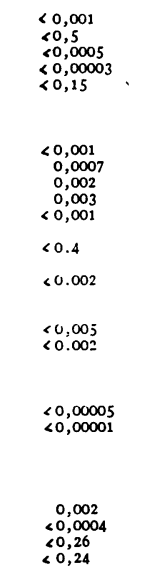 \\
\hline
\end{tabular}

chimique donnant une concentration supérieure si elle ne tient pas compte de la forme chimique où se trouve l'élément.

Le traitement de ce matériau sous plasma argonhydrogène-oxygène a été effectué avec 2 concentra- tions différentes d'oxygène $(1 \%$ d'hydrogène et $0,1 \%$ puis $0,01 \%$ d'oxygène).

L'analyse du matériau par ESCA après traitement a été faite sur la face supérieure de la queue de l'échantillon, c'est-à-dire à un endroit où l'ensemble des impuretés éliminées de la partie purifiée se sont concentrées.

Le résultat de l'analyse montre (Fig. 6) :

1) que la concentration en bore lié au silicium diminue lorsque la concentration en oxygène

Tableau V. - Résultats de la spectrométrie de masse par étincelle. Echantillon de silicium métallurgique purifié par F.Z.P. (4 passages; $40 \mathrm{~cm} / \mathrm{h}$ ), traitement acide). Résultats en ppm poids.

[Analysis by mass spectrometry. Sample of metallurgical grade silicon purified by plasma zone melting ( 4 pass, $40 \mathrm{~cm} / \mathrm{h}$ ), acidic leaching.]

$\begin{array}{ll}\text { B } & 0,15 \\ \mathrm{C} & 0,08-0,7 \\ \mathrm{~F} & 0,02 \\ \mathrm{Mg} & 0,04 \\ \mathrm{Al} & 0,1 \\ \mathrm{P} & 0,1 \\ \mathrm{~S} & <0,4 \\ \mathrm{Cl} & 0,09-0,9 \\ \mathrm{~K} & 0,3 \\ \mathrm{Cd} & 0,08 \\ \mathrm{Ti} & <0,08 \\ \mathrm{~V} & <0,08 \\ \mathrm{Cr} & <0,1 \\ \mathrm{Cu} & 0,35 \\ \mathrm{Zn} & <0,2 \\ \mathrm{Ga} & <0,2\end{array}$


augmente. Cette mesure faite en queue de barreau permet :

- de prévoir que les espèces $\mathrm{Si}_{x} \mathrm{~B}_{y}$ de départ sont décomposées sous l'effet thermique du plasma d'une part puis oxydées sous forme de $\mathrm{SiO}_{2}$ et $\mathrm{B}_{x} \mathrm{O}_{y}$ d'autre part pour être enfin drainées en queue de barreau,

- de mieux expliquer l'augmentation de la résistivité du silicium dans la partie purifiée du barreau comme nous l'avons montré précédemment,

- enfin, la surface des pics ESCA du bore lié au silicium diminue d'un facteur 2 entre l'échantillon de départ et celui traité par un plasma contenant
$0,1 \% \mathrm{~d}^{\prime} \mathrm{O}_{2}$. Ce résultat est tout à fait en accord avec le bilan matière calculé à partir des mesures de résistivité.

2) Avec l'augmentation de la concentration en oxygène dans le plasma les oxydes $\mathrm{BO}, \mathrm{B}_{2} \mathrm{O}_{2}$ ont des concentrations croissantes. Ces oxydes en quantité très importante en queue du barreau confirment l'hypothèse du drainage du bore sous forme d'oxyde. Par ailleurs le bore oxydé sous forme $\mathrm{B}_{2} \mathrm{O}_{3}$ correspondant au photoélectron d'énergie $191,8 \mathrm{eV}$ est absent. Cet oxyde serait donc éliminé sous l'effet thermique du plasma.

Tableau VI. - Analyse du silicium par spectrométrie d'émission plasma. Concentration dans l'échantillon solide en ppb poids $\left(10^{-9}\right)$.

[Analysis by atomic emission spectroscopy from inductively coupled plasma. Concentration of the solid sample in ppb weight $\left(10^{-9}\right)$.]

\begin{tabular}{|c|c|c|c|c|c|}
\hline Elements & $\begin{array}{c}1 \\
\substack{\text { siletum } \\
\text { monocristal1in }}\end{array}$ & 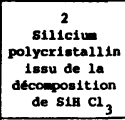 & 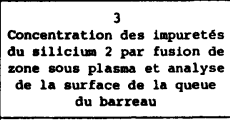 & \begin{tabular}{|c|} 
\\
Silicium polycristallin \\
obtenu par fuston de \\
zone sous plasma d \\
partir du silicium \\
métallurglque 99,7 \\
\end{tabular} & \begin{tabular}{|l} 
Concentration des impuretés \\
du sillcilum 4 par fusion de \\
zone sous plasas ot analyse \\
de la surface de la queue \\
du barreau
\end{tabular} \\
\hline c & $2 \pm 0,3$ & $16 \pm 4$ & $29 \pm 5$ & $5 \pm 0,5$ & $104 \pm 10$ \\
\hline $\mathrm{cu}$ & $1 \pm 0,5$ & $43 \pm 2$ & $307 \pm 10$ & $2 \pm 0,5$ & $116 \pm 4$ \\
\hline $\mathrm{Mn}_{\mathrm{n}}$ & $5 \pm 1$ & $45 \pm 3$ & $59 \pm 3$ & $<0,6$ & $170 \pm 6$ \\
\hline Al & $\leqslant 0,3$ & $<0,3$ & $11 \pm 3$ & $<0,6$ & $25 \pm 5$ \\
\hline B & $35 \pm 20$ & $35 \pm 20$ & $985 \pm 100$ & $55 \pm 30$ & $6985 \pm 100$ \\
\hline
\end{tabular}

3) Le bore métallique présent en queue du barreau est le même que dans l'échantillon de départ. Celui-ci sous l'effet du plasma s'oxyde sous une forme (BO, $\mathrm{B}_{2} \mathrm{O}_{2}, \mathrm{BO}_{3}$ ou $\mathrm{B}_{2} \mathrm{O}_{3}$ ) puis drainé en queue de barreau. Les atomes de bore métallique mesurés en queue correspondraient donc à un oxyde autre que $\mathrm{B}_{2} \mathrm{O}_{3}$, décomposés sous l'effet thermique du plasma.

4) A partir de la figure 6 il est possible d'indiquer les concentrations respectives du bore sous différentes formes. En effet dans cette analyse il s'agit des photoélectrons $B_{1 \mathrm{~s}}$ émis des échantillons à analyser, les surfaces de chaque pic sont donc comparables sans facteur correctif. En admettant que les 74 ppm de bore considérés dans l'échantillon de départ concerne la liaison dopante active Si-B, le tableau VII présente l'évolution des concentrations en bore libre, en bore lié à l'oxygène et en bore lié au silicium. L'échantillon témoin aurait $430 \mathrm{ppm}$ de bore total dont $74 \mathrm{ppm}$ de bore actif. Après traitement sous plasma oxygéné on constate alors la bonne corrélation entre la diminution de la concentration en bore lié au silicium et la résistivité d'une part et l'importance de la concentration des oxydes en queue de barreau d'autre part.

Des analyses du $\mathrm{Cu}_{3 \mathrm{~s}}, \mathrm{P}_{2 \mathrm{p}}$ et $\mathrm{O}_{1 \mathrm{~s}}$ ont été également faites sur ces échantillons traités sous plasma oxygéné. Il apparaît que de la même façon que dans le cas du bore ces éléments se concentrent en queue de barreau avec une concentration croissante lorsque la teneur en oxygène du plasma augmente (Fig. 7).
Tableau VII. - Concentration en ppm poids des différentes formes de bore dans le silicium de départ et dans le silicium traité en plasma oxygéné. Hypothèse : B-Si $=74$ ppm.

[Concentration of different forms of bounding between boron and silicon or oxygen in untreated sample, sample treated by plasma with $0.01 \%$ of $\mathrm{O}_{2}$ and $0.1 \%$ of $\mathrm{O}_{2}$.]

\begin{tabular}{|c|c|c|c|c|}
\hline B-Si & B & BO Ou $\mathrm{B}_{2} \mathrm{O}_{2}$ & $\mathrm{BO}_{3}$ & TOTAL \\
\hline 74 & 74 & 282 & 0 & $430 \mathrm{ppm}$ \\
44 & 74 & 444 & 242 & $804 \mathrm{pPm}$ \\
15 & 74 & 713 & 497 & $1299 \mathrm{ppm}$ \\
\hline
\end{tabular}

Une analyse de la queue d'un barreau de silicium métallurgique traité en plasma argon $-1 \%$ hydrogène et $0,1 \%$ d'oxygène permet de préciser que l'oxyde de silicium est également présent en surface mais en faible concentration et que l'oxyde formé se concentre sur cette surface puisque sa concentration à cœur est beaucoup plus faible (Fig. 8).

3.4.2 Analyse par infrarouge. - L'analyse par spectrométrie d'absorption infrarouge à température ambiante d'un échantillon de silicium monocristallin $(1-3 \Omega . \mathrm{cm})$ indique (Fig. 9) la présence d'une bande de vibration $\mathrm{Si}_{2} \mathrm{O}$ à $1106 \mathrm{~cm}^{-1}$. Cet échantillon 


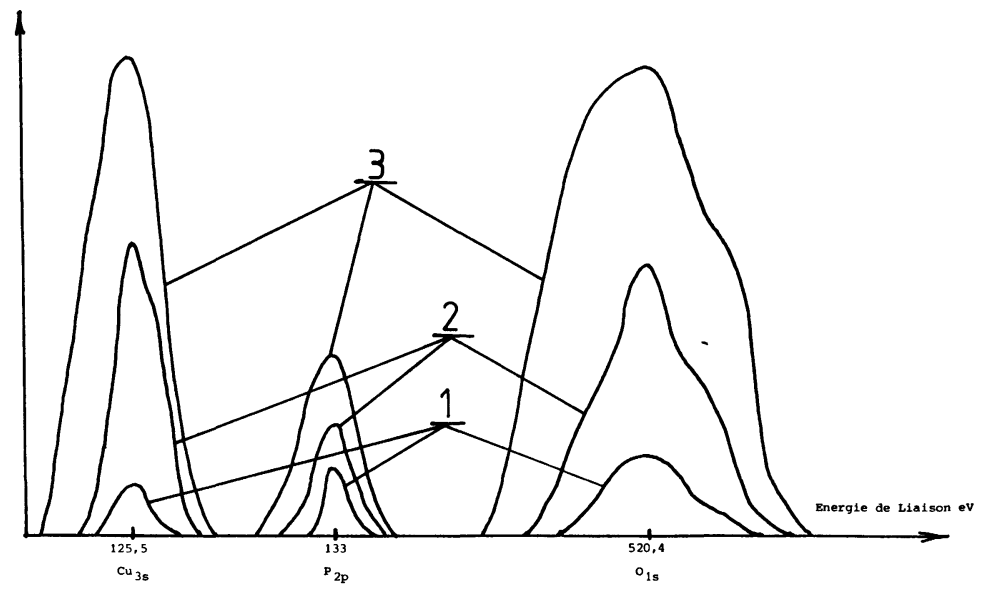

Fig. 7. - Analyse ESCA du silicium électronique $\mathrm{P}^{+}$. Mise en évidence du drainage des impuretés phosphore et cuivre en queue du barreau. Influence de la concentration en oxygène du plasma $\left(\mathrm{Ar}+1 \% \mathrm{H}_{2}\right)$ sur le drainage de ces impuretés dans le laitier. 1) Echantillon témoin Si électronique $\mathrm{P}^{+}$. 2) Analyse de la surface de la queue après traitement avec $0,01 \% \mathrm{~d}^{\prime} \mathrm{O}_{2}$. 3) Analyse de la surface de la queue après traitement avec $0,1 \% \mathrm{~d}^{\prime} \mathrm{O}_{2}$.

[ESCA analysis of Pt electronic silicon. Demonstrate of the drainage of impurities in the tail of the bar such as phosphorus and copper. Influence of oxygen concentration in plasma (argon $+1 \%$ of hydrogen) on the drainage of that impurities in the slag. 1) Untreated sample : Pt electronic silicon. 2) Analysis of the surface of the tail after treatment with $0.01 \%$ of $\mathrm{O}_{2}$. 3) Analysis of the surface of the tail after treatment with $0.1 \%$ of $\mathrm{O}_{2}$.]

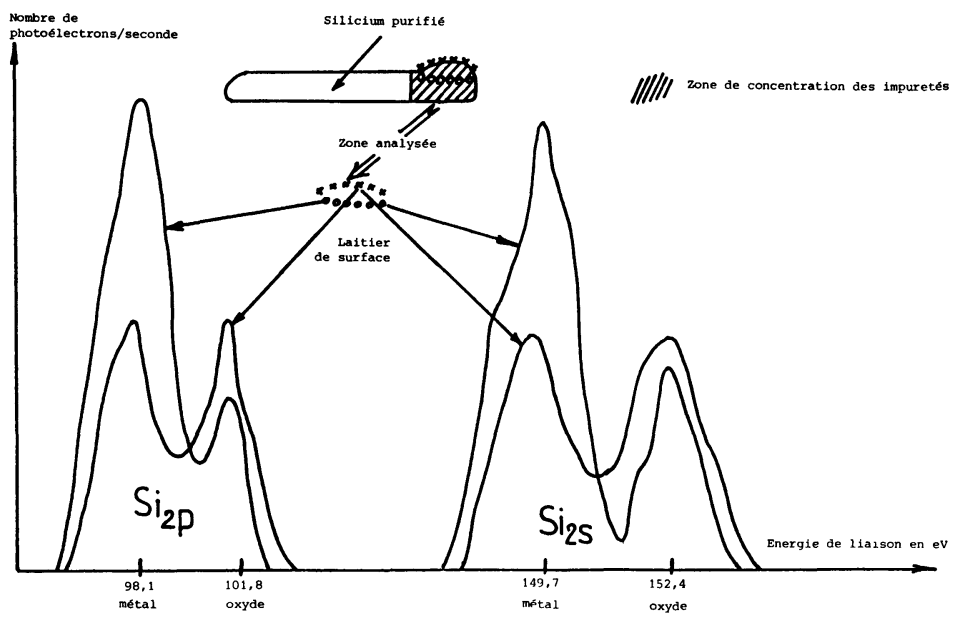

Fig. 8. - Analyse ESCA : Echantillon de silicium métallurgique purifié par fusion de zone sous plasma réactif (argon + $1 \% \mathrm{H}_{2}+0,1 \% \mathrm{O}_{2}$ ). Analyse du laitier de surface. Mise en évidence de la ségrégation de l'oxyde en surface.

[ESCA analysis : metallurgical grade silicon purified by plasma zone melting process with a reactive plasma $\left(\operatorname{argon}+1 \% \mathrm{H}_{2}\right.$ $+0.1 \% \mathrm{O}_{2}$ ). Analysis of the slag. Demonstrate of the segregation of the oxide on the surface.]

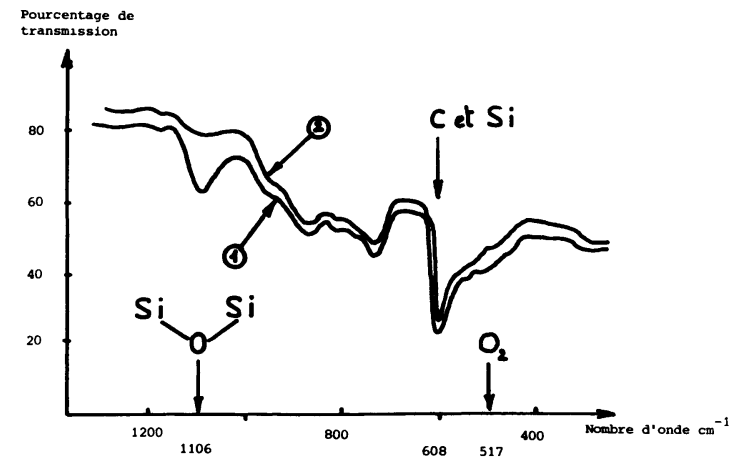

Fig. 9. - Analyse par spectrométrie de transmission en IR : 1) Dosage du silicium monocristallin témoin $(3 \Omega . \mathrm{cm})$. 2) Dosage du silicium monocristallin témoin $(3 \Omega . \mathrm{cm})$ traité par un plasma argon $+1 \% \mathrm{~d}^{\prime} \mathrm{H}_{2}$.

[Characterization of oxygen in silicon by infrared absorption by transmission spectroscopy. 1) Untreated mono silicon sample $(3 \Omega . \mathrm{cm})$. 2) Sample treated by plasma $\left(\operatorname{argon}+1 \% \mathrm{H}_{2}\right)$.] 
soumis à une fusion de zone sous plasma argon $+1 \%$ d'hydrogène provoque dans la partie purifiée la disparition de cette bande alors que, celle de l'oxygène à $517 \mathrm{~cm}^{-1}$ et celle du carbone à $608 \mathrm{~cm}^{-1}$ qui se superpose à une bande de vibration intrinsèque du silicium, sont toujours visibles. Ceci indique donc que l'on contrôle parfaitement la concentration en oxygène dans l'échantillon de silicium purifié sous plasma et confirme d'une part la possibilité de diminuer la concentration en oxyde et d'autre part que la pollution par l'oxygène lors du traitement est inexistante.

Dans le cas où l'échantillon de silicium monocristallin $(1-3 \Omega . \mathrm{cm})$ est soumis au traitement par fusion de zone sous plasma argon $+1 \%$ d'hydrogène $+0,1 \%$ d'oxygène, l'analyse par infrarouge montre que la concentration en oxyde et en oxygène dans la partie purifiée n'a pas variée.

De l'analyse par spectrométrie d'absorption infrarouge, il ressort que la concentration en oxygène de l'échantillon traité est parfaitement maîtrisée et peut être contrôlée par la composition chimique du fluide plasmagène.

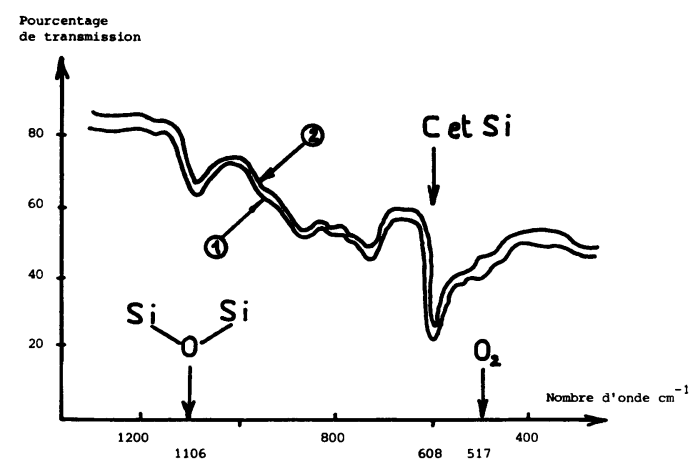

Fig. 10. - Analyse par spectrométrie de transmission en IR : 1) Dosage du silicium monocristallin témoin $(3 \Omega . \mathrm{cm})$. 2) Dosage du silicium monocristallin témoin $(3 \Omega . \mathrm{cm})$ traité par un plasma argon $+1 \% \mathrm{H}_{2}+0,1 \% \mathrm{~d}^{\prime} \mathrm{O}_{2}$.

[Characterization of oxygen in silicon by infrared absorption by transmission spectroscopy. 1) Untreated monocrystalline silicon sample $(3 \Omega . \mathrm{cm}), 2)$ Sample treated by plasma with argon $+1 \% \mathrm{H}_{2}+0.1 \% \mathrm{O}_{2}$.]

3.5 Conclusion. - Il apparaît que l'élément clef de l'élimination du bore est l'introduction d'oxygène dans le plasma d'argon-hydrogène.

Cette élimination est favorisée par la réactivité chimique du plasma oxygéné et par l'action thermique de celui-ci. La combinaison de ces deux effets provoque une évaporation du composé complétee d'une concentration en queue de barreau.

Une étude thermodynamique permet de proposer une interprétation du processus chimique à partir des résultats expérimentaux.

4. Interprétations des résultats à partir des données thermodynamiques. - 4.1 INTRODUCTION. - L'étude expérimentale confirme qu'une partie du bore présent est éliminée par drainage à la surface et en queue de barreau, l'autre partie est éliminée sous forme d'un composé volatil. Le drainage du bore en queue de barreau est le résultat du déplacement d'un composé du bore dans la goutte liquide, mais son élimination en surface apparaît comme un aspect à la fois thermique et chimique qui est bien spécifique de la technique plasma.

Le rôle du plasma argon- $\mathrm{H}_{2}-\mathrm{O}_{2}$ sur le processus d'élimination du bore exige une étude de la chimie du bore afin d'identifier la nature des espèces susceptibles d'être produites; trois types de composés peuvent apparaître : les boranes, les borures de silicium et les oxydes de bore [19].

4.2 LeS Boranes. - Il existe deux catégories de borane : les hydrures stables $\mathrm{B}_{n} \mathrm{H}_{n+4}$ et les hydrures instables $\mathrm{B}_{n} \mathrm{H}_{n+6}$. Lorsque la température augmente il $\mathrm{y}$ a perte d'hydrogène et ils sont complètement décomposés à $250^{\circ} \mathrm{C}$. Dans les conditions thermiques de la fusion par plasma le bore n'est donc pas éliminé sous forme d'hydrure de bore.

4. 3 Les BORURES DE SILICIUM. - Ces composés $\left(\mathrm{B}_{3} \mathrm{Si}, \mathrm{B}_{4} \mathrm{Si}, \mathrm{B}_{6} \mathrm{Si}, \mathrm{B}_{12} \mathrm{Si}\right)$ se forment à haute température $(1800-2500 \mathrm{~K})$ et sont très stables. Toutefois en surface ils pourront être éliminés par décapage par le mélange acide $\left(\mathrm{HF}-\mathrm{HNO}_{3}\right)$ à condition de ne pas avoir été détruit par l'oxygène du plasma.

4. 4 LES OXYDES DE BORE. - Les oxydes de bore susceptibles de se former sont nombreux ( $\mathrm{BO}, \mathrm{BO}_{3}$, $\mathrm{B}_{2} \mathrm{O}_{2}, \mathrm{~B}_{4} \mathrm{O}_{3}, \mathrm{~B}_{3} \mathrm{O}_{2}, \mathrm{~B}_{4} \mathrm{O}_{5}$ et $\mathrm{B}_{2} \mathrm{O}_{3}$ ). Dans les conditions de température de l'expérience l'oxyde le plus stable est l'anhydride borique $\mathrm{B}_{2} \mathrm{O}_{3}$ (point de fusion à $580^{\circ} \mathrm{C}$, point d'ébullition $2400^{\circ} \mathrm{C}$ ). La stabilité thermodynamique de $\mathrm{B}_{2} \mathrm{O}_{3}$ est supérieure à celle de $\mathrm{SiO}$ et $\mathrm{SiO}_{2}[20]$ de sorte qu'en présence d'oxygène $\mathrm{B}_{2} \mathrm{O}_{3}$ se forme préférentiellement à tous les autres oxydes. En surface du silicium fondu sous plasma la température atteinte est proche de $2400{ }^{\circ} \mathrm{C}$ ainsi $\mathrm{B}_{2} \mathrm{O}_{3}$ pourra se vaporiser.

L'analyse ESCA permet d'ailleurs de mettre en évidence l'absence de $\mathrm{B}_{2} \mathrm{O}_{3}$ en surface de l'échantillon traité. Ceci confirme bien les considérations thermodynamiques énoncées précédemment. Cette analyse montre par ailleurs que l'oxyde de silicium est également présent en surface des échantillons mais en faible concentration et que l'oxyde formé se concentre en surface puisque sa concentration à cœur est très faible.

4.5 CONCLUSION SUR L'ÉLIMINATION DU BORE. L'étude thermodynamique et expérimentale suivie d'analyse IR et ESCA indique qu'en présence d'oxygène dans le plasma l'élimination du bore est réalisée d'une part par évaporation de $\mathrm{B}_{2} \mathrm{O}_{3}$ et d'autre part par drainage d'oxydes de bore en queue de barreau. L'hydrogène présent dans le plasma permet d'augmenter le transfert thermique et d'augmenter ainsi la température de la surface du silicium liquide, favorisant alors l'évaporation de $\mathrm{B}_{2} \mathrm{O}_{3}$. 
5. Elimination du bore dans le silicium métallurgique : rôle du laitier de surface. - Nous avons montré comment le bore pouvait être extrait d'une matrice de silicium ultra-pur. Nous nous proposons maintenant d'étudier l'élimination du bore dans un silicium métallurgique industriel contenant selon les cas de 0,1 à $2 \%$ d'impuretés. Un silicium métallurgique industriel contient 20 à $40 \mathrm{ppm}$ de bore et d'autres impuretés en concentration plus importante. Ces impuretés réagissent également avec l'oxygène du plasma et se concentrent en surface pour former un laitier dont les propriétés physico-chimiques conduisent à un piégeage des impuretés et notamment du bore.

5. 1 RÉSUltats EXPÉRIMENTAUX. - Selon le soin apporté au choix du sable de départ et des réducteurs, le silicium métallurgique titre $98 \%$ à $99,9 \%$ de' silicium (Tableau III). Afin de pouvoir conclure sur le rôle du matériau de départ nous avons traité par fusion de zone sous plasma deux silicium métallurgiques. L'opération a lieu sous plasma argon-hydrogène-oxygène, nous effectuons 4 passages à $40 \mathrm{~cm} / \mathrm{h}$, et un décapage de la surface par un mélange acide $\left(\mathrm{HF}-\mathrm{HNO}_{3}\right.$ ) entre chaque passage.

Les analyses par radioactivation (Tableau IV) des matériaux purifiés montrent que dans le cas du silicium métallurgique 1 la concentration globale en impureté est voisine de $2 \mathrm{ppm}$. Pour le silicium métallurgique 2 la concentration globale après traitement est voisine de $0,2 \mathrm{ppm}$.

Une analyse par spectrométrie de masse à étincelle (Tableau V) du matériau purifié issu du silicium métallurgique 2 complète les informations obtenues par radioactivation. Nous constatons que le bore est passé de 40 ppm à 0,15 ppm et que les autres impuretés ont toutes une teneur inférieure au ppm. Comme nous l'avions fait remarquer les limites de détection sont moins bonnes en spectrométrie de masse à étincelle qu'en radioactivation et pour compléter cette analyse chimique nous avons effectué des analyses par spectrométrie d'émission plasma (Tableau VI).

La sensibilité de cette technique analytique nous a permis de doser et de comparer trois silicium ultrapurs : le silicium polycristallin Wacker, le silicium monocristallin et le silicium de fusion de zone sous plasma. Les résultats indiquent qu'il s'agit de matériaux de pureté voisine et confirme par ailleurs l'élimination du bore dans le silicium métallurgique par fusion de zone sous plasma réactif. Néanmoins les limites de détection ont été atteintes pour le manganèse et l'aluminium. Afin de pouvoir comparer les teneurs résiduelles nous avons effectué un nouveau traitement par fusion de zone sous plasma dans les mêmes conditions sur la partie pure de l'échantillon. L'opération avait pour but de concentrer les impuretés restantes de l'échantillon à la surface de la queue de celui-ci et d'analyser la solution de dissolution de la queue du barreau, zone où les impuretés s'étaient concentrées. Les résultats n'ont donc pas une valeur quantitative stricte mais permettent d'effectuer une comparaison entre le silicium polycristallin Wacker et le silicium de fusion de zone sous plasma (Tableau VI).

Les analyses indiquent que sur des échantillons où la teneur de départ en bore est très faible respectivement 35 et $55 \mathrm{ppb}$ le traitement par fusion de zone sous plasma réactif conduit à un drainage du bore en surface puis en queue du barreau et ceci confirme bien les hypothèses émises précédemment sur le silicium monocristallin ultra-pur.

Les mesures ESCA indiquent qu'en présence d'oxygène, le bore, sous forme d'oxyde, se concentre en surface avec $\mathrm{SiO}_{2}$ (Fig. 6). Par ailleurs une analyse par rayon $\mathrm{X}$ de cette surface en queue de barreau révèle un composé de type $\mathrm{FeSi}_{2}$.

Ce laitier de structure physico-chimique complexe est donc vraisemblablement à l'origine de la cinétique d'élimination du bore. Afin de mieux comprendre ceci nous pouvons analyser la réactivité chimique du bore et tenter de donner une interprétation à la purification par fusion de zone sous plasma réactif.

\section{3 RÔLE DU LAITIER SUR L'ÉLIMINATION DU BORE. -} Une étude bibliographique effectuée par Pastor [19] indique combien la réactivité chimique du bore est importante. Les composés les plus importants pouvant apparaître peuvent se diviser en plusieurs groupes : les nitrures, les carbures et les borures. Les nitrures et carbures de bore n'ont vraisemblablement qu'un rôle secondaire dans notre cas par contre les borures peuvent se former dans un milieu oxydant. Les borures fondent sans décomposition à des températures très élevées [19] et leurs points de fusion sont supérieurs à $2000^{\circ} \mathrm{C}$. Ces composés sont réputés bons conducteurs électriques. C'est ainsi que $\mathrm{TiB}_{2}$ a une résistivité comparable à celle du fer. Cette remarque montre que bien que le titane soit considéré comme:‘tueur d'électron il aura des propriétés électriques très différentes s'il se trouve à l'état libre ou combiné dans le silicium; la résistivité globale du matériau en sera donc affectée.

Les borures ont une grande inertie chimique vis-àvis des réactifs non oxydants. Par contre à haute température, ils s'oxydent. Dans le silicium métallurgique traité par fusion de zone sous plasma réactif le bore peut se combiner avec les impuretés et ainsi être éliminé. Tout ceci confirme bien le fait qu'on ne peut considérer l'influence d'une impureté sur l'effet photovoltaïque comme indépendante des autres impuretés. Ainsi dans le barreau de silicium purifié les impuretés restantes ne sont pas à l'état libre uniquement mais combiné également sous forme de microprécipités [21-24]. Ainsi la réactivité chimique du bore en milieu oxydant à haute rempérature peut être utilisée pour son élimination. Le mode opératoire retenu conduit donc à la formation d'un laitier en surface ayant des propriétés physico-chimiques responsables de l'élimination du bore. 
5.3.2 RÔle DU LAITIER OBTENU PAR UN PLASMA OXYGÉNÉ. - De nombreux composés oxygénés sont susceptibles de se former en cours de traitement. Certains ont été cités dans la bibliographie [19] et présentent la particularité d'être stables et leur composition se rapprochant des compositions des laitiers synthétiques utilisés en métallurgie extractive, on trouve les systèmes binaires ou ternaires suivants :

$$
\begin{aligned}
& \mathrm{SiO}_{2}-2 \mathrm{Na}_{2} \mathrm{O} \\
& \mathrm{CaO}-\mathrm{SiO}_{2} \\
& \mathrm{Na}_{2} \mathrm{O}-\mathrm{B}_{2} \mathrm{O}_{3}-\mathrm{SiO}_{2} \\
& \mathrm{CaO}-\mathrm{Al}_{2} \mathrm{O}_{3}-\mathrm{SiO}_{2}
\end{aligned}
$$

La chimie de ces complexes oxydés est considérée comme délicate néanmoins à partir des données physico-chimiques des chercheurs s'en sont servi pour purifier le silicium métallurgique [25-26].

La purification par laitier synthétique est une extraction liquide-liquide et le choix du laitier purificateur doit répondre à plusieurs exigences :

1) être non missible avec le silicium liquide,

2) avoir un point de fusion pas trop éloigné du silicium,

3) ne pas contaminer le silicium fondu,

4) avoir une grande affinité chimique avec les impuretés que l'on souhaite éliminer.

Les oxydes suivants répondaient à ces exigences et leur utilisation [25] a effectivement montré leur aptitude à purifier le silicium. Il s'agit de

$$
\begin{aligned}
& \mathrm{CaO}-\mathrm{SiO}_{2} \\
& \mathrm{CaO}-\mathrm{MgO}-\mathrm{SiO}_{2} \\
& \mathrm{CaO}-\mathrm{BaO}-\mathrm{SiO}_{2} \\
& \mathrm{BaO}-\mathrm{MgO}-\mathrm{SiO}_{2}
\end{aligned}
$$

On comprend donc mieux l'intérêt d'utiliser un plasma oxydant pour l'élaboration de silicium photovoltaïque qui permet ainsi d'obtenir un laitier ayant une composition complexe se composant de silice et d'oxydes des impuretés du silicium métallurgique ( $\mathrm{Ca}, \mathrm{Al}, \mathrm{C}, \mathrm{Ba}, \mathrm{Mg}, \mathrm{Fe}, \mathrm{B} . .$. etc.).

Celui-ci a donc la faculté de piéger une impureté telle que le bore sous forme d'oxyde et ainsi d'élaborer un matériau de haute pureté avec une cinétique d'élimination très grande. En effet la purification n'est plus contrôlée par la diffusion comme en fusion de zone classique mais par la convection. Cette convection est d'autant plus forte que la température est élevée en surface par la présence du plasma et la viscosité du silicium à cette température $2400^{\circ} \mathrm{C}$ est inférieure à celle de l'eau à $50^{\circ} \mathrm{C}$. Par ailleurs l'augmentation de la température en surface diminue la viscosité du laitier qui a alors ses propriétés purificatrices qui s'améliorent. Les impuretés sont donc piégées par le laitier en surface et drainées en queue de barreau. Une partie de l'oxyde formée sera vaporisée, l'autre partie drainée pourra être éliminée par le décapage de la surface par $\mathrm{HF}-\mathrm{HNO}_{3}$.

6. Conclusion. - L'ensemble des moyens analytiques nous ont permis de mettre en évidence l'élimination du bore par la technique de fusion de zone sous plasma réactif. L'interaction du plasma oxygéné avec la surface du silicium est à l'origine de l'élimination du bore. Les échantillons de silicium électronique dopés au bore traité par plasma soulignent le rôle de la teneur en oxygène dans le plasma pour éliminer le bore.

L'ESCA nous permet de conclure sur la nature des liaisons créées entre le bore et l'oxygène et de montrer que $\mathrm{B}_{2} \mathrm{O}_{3}$ est vaporisé. La spectrométrie d'absorption infrarouge montre qu'il n'y a pas d'oxygène résiduel dans l'échantillon purifié par le plasma oxygéné.

Dans le cas du silicium métallurgique le plasma oxygéné génère un laitier en surface de la zone fondue. Ce laitier est à l'origine du piégeage du bore et augmente par ailleurs la cinétique de purification.

Sur le tableau II nous avons porté (colonne 5) les résultats des mesures électriques effectuées sur un échantillon de silicium métallurgique $(99,7 \%)$ purifié par plasma. Le rendement photovoltaïque d'un tel échantillon est de 3,7\%. Sur un échantillon de grande taille de type industriel et avec une couche anti-reflet le rendement photovoltaïque serait de $6 \%$. Par ailleurs nous savons que le choc thermique est important avec le plasma et ceci provoque une perturbation cristallographique. Ce rendement photovoltaïque serait amélioré en complétant le traitement par une fusion contrôlée, celui-ci pourrait vraisemblablement dépasser $10 \%$.

\section{Bibliographie}

[1] Durand, H., Philos. Trans. R. Soc. A 295 (1980) 435-443.

[2] Rapport interne : Wacker-Chemitronic GmbH, 1979. 10-20, A type of Large-area polycristalline silicon with colummar structure for high-efficiency terrestrial solar cells.

[3] Sirtl, E., Rapport interne : Wacker-Heliotronic $\mathrm{GmbH}$, Technology and economics of starting materials for low-cost silicon solar cells.
[4] Hill, D. E., Gutsche, H. W., Wang, M. S., Cupta, K. P., Tucker, W. F., Dowdy, J. D., Crepin, R. J., 12th IEEE Photovoltaic spec. conf., 1518 nov. 1976, Bâton-Rouge-Louisiana, U.S.A.

[5] Morvan, D., Amouroux, J., Revel, G., Revue Phys. Appl. 15 (1980) 1229.

[6] Amouroux, J., Morvan, D., Revel, G., Fedoroff, M., Rouchaud, J. C., Revue Phys. Appl. 15 (1980) 1239. 
[7] LINDMAYER, J., Rapport interne, 1978, Solar corporation Rockville, Maryland, Characteristics of semicrystalline silicon solar cells.

[8] Kuper, A. B., Rapport interne, 1978, Case Western Reserve University Cleveland (Work performed at NASA, Lewis Research Center), Poly and single crystal silicon solar cells.

[9] Huber, D., Wahlich, R., Helmreich, D., Rapport interne, 1978, Wacker-Chemitronic and Heliotronic $\mathrm{GmbH}$, Typical properties of multicrystalline silicon material.

[10] Ghosh, A. D., Fishman, C., Feng, T., J. Appl. Phys. 51 (1), January 1980.

[11] MaisSel, L. I., Hand book, chap. 18.

[12] Bean, K. E., Hentzchel, M. P., Colman, D., J. Appl. Phys. 40 (1969) 2358.

[13] Roy, K., Rapport interne, AEG, Telefunken Serienprodukte Ag Theresienstr. 2, 7100 Heilbronn. Electrical characterization of non single crystalline silicon.

[14] Valdes, L. B., Proc. I.R.E. 42 no 2 (1954) 420.
[15] Pauleau, Y., Actualité Chimique (1981) 7.

[16] PAJot, B., Analysis v.s., no 7 (1977) 293-303.

[17] Groue, A. S., Leistiko, O., SaH, I. R., Sah, C. T., J. Appl. Phys. 35 no 9 (1964) 2695-2701.

[18] Theurer, H. C., J. Met. Transaction AIME (1956) 1316-1319.

[19] Pastor, H. et Thevenot, F., Inf. Chim. no 178.

[20] Thermochemical Tables JANAF, Second Edition, StUll, D. R., Prophet, H., June 1971.

[21] Brevet, Aluminium Company of America, no 8011626 du 23 mai 1980.

[22] Brevet Montedison S.p.A. (Italie) no 7927338 du 6 novembre 1979.

[23] Brevet Allemand no 2924584 du 15 janvier 1981.

[24] Brevet, The General Trusee Company INC (Suisse) n $^{\circ} 1469486$ du 14 septembre 1965 .

[25] Secco d'aragona, F., Liaw, H. M., Heminger, D. M., Proceedings of the symposium on : Materials and New processing technologies for photovoltaics. The Electrochemical Society, volume 81-3, p. 119.

[26] Pizzini, S., Sol. Energy Mat. 6 (1982) 253-297.

[27] Wolf, S. C. (Wiley Interscience) 537, 311-33. 